MATHEMATICS OF COMPUTATION

Volume 72, Number 244, Pages 2001-2012

S 0025-5718(03)01577-1

Article electronically published on May 23, 2003

\title{
CODING THE PRINCIPAL CHARACTER FORMULA FOR AFFINE KAC-MOODY LIE ALGEBRAS
}

\author{
M. K. BOS
}

\begin{abstract}
In this paper, an algorithm for computing the principal character for affine Lie algebras is discussed and presented. The principal characters discovered using this program are given and/or proven. Results include level 2 and 3 character formulas in $A_{2 n-1}^{(2)}$ and the sole existence of the RogersRamanujan products in $A_{1}^{(1)}, A_{2}^{(1)}, A_{2}^{(2)}, C_{3}^{(1)}, F_{4}^{(1)}, G_{2}^{(1)}, A_{7}^{(2)}$.
\end{abstract}

\section{IntRoduction}

The principal character of an affine Kac-Moody Lie algebra (see 4]) is an extremely powerful tool for understanding the structure of its integrable highest weight modules. The Rogers-Ramanujan products occur in the character formulas of several affine Lie algebras: $A_{1}^{(1)}, A_{2}^{(1)}, A_{2}^{(2)}, A_{7}^{(2)}, C_{3}^{(1)}, F_{4}^{(1)}$, and $G_{2}^{(1)}$. Using the Rogers-Ramanujan identities, the realizations of these representations have all been constructed in [2], 3], [5], 7], [8, 9], 11], [12], and [14].

The occurrence of the Rogers-Ramanujan products in the character formulas significantly simplifies the construction of the corresponding modules. Furthermore, we can go the other way. Lepowsky and Wilson gave a Lie theoretic proof ([] $)$ of the Rogers-Ramanujan identities using their vertex operator realization of $A_{1}^{(1)}$ (5]). A program which reliably calculates the principal character formula is useful in constructing the associated modules. In this paper, an algorithm is presented for this calculation and several formulas discovered using this program are proven. Maple code is available via email or on the web at http://it.stlawu.edu/ mbos/ research/character.html.

\section{Preliminaries}

We let $\mathfrak{g}=\mathfrak{g}(A)$ be an affine Lie algebra with $3(n+1)$ generators $e_{i}, f_{i}$, and $h_{i}$ associated with a symmetrizable generalized Cartan matrix (GCM), $A=\left(a_{i j}\right)_{i, j=0}^{n}$, and let $d$ be a derivation on $\mathfrak{g}$. Furthermore, we define the semidirect products $\tilde{\mathfrak{g}}=\mathfrak{g} \oplus \mathbf{C} d$ and $\tilde{t}=t \oplus \mathbf{C} d$, abelian subalgebras, where $t=\operatorname{span}\left\{h_{0}, \ldots, h_{n}\right\}$. For all $0 \neq \alpha \in \tilde{t}^{*}$, we define

$$
\mathfrak{g}_{\alpha}=\{x \in \mathfrak{g} \mid[h, x]=\alpha(h) x, \forall h \in \tilde{t}\} .
$$

Received by the editor October 3, 1999 and, in revised form, March 27, 2002.

2000 Mathematics Subject Classification. Primary 17B67, 17B10.

Key words and phrases. Affine Lie algebra, principal character.

(C)2003 American Mathematical Society 
Then, $\Delta=\left\{\alpha \in \tilde{t}^{*} \mid \alpha \neq 0\right.$ and $\left.\mathfrak{g}_{\alpha} \neq(0)\right\}$ is the set of roots. The simple roots, $\Phi=\left\{\alpha_{0}, \ldots, \alpha_{n}\right\} \subseteq \Delta$, are defined by the condition

$$
e_{i} \in \mathfrak{g}_{\alpha_{i}} .
$$

$\Phi$ is linearly independent and $\Delta$ can be broken down into positive and negative roots, as follows:

$$
\Delta_{+}=\left\{\alpha \in \Delta \mid \alpha=\sum_{i=0}^{n} c_{i} \alpha_{i}, c_{i} \geq 0\right\}
$$

and

$$
\Delta_{-}=\left\{\alpha \in \Delta \mid \alpha=\sum_{i=0}^{n} c_{i} \alpha_{i}, c_{i} \leq 0\right\}
$$

where $\Delta=\Delta_{+} \cup \Delta_{-}$.

The simple reflections, $r_{i}$, are defined by

$$
r_{i}(\beta)=\beta-\beta\left(h_{i}\right) \alpha_{i}, \quad i=0, \ldots, n .
$$

The Weyl group, $\mathrm{W}$, is the group generated by $r_{0}, \ldots, r_{n}$. Now $\Delta$ can be broken down in another useful way, real and imaginary roots:

$$
\Delta^{\mathrm{re}}=\left\{\alpha \in \Delta \mid \exists w \in W \text { such that } w \alpha=\alpha_{i}, \text { for some } i\right\}
$$

and

$$
\Delta^{\mathrm{im}}=\left\{\alpha \in \Delta \mid \alpha \notin \Delta^{\mathrm{re}}\right\} .
$$

Clearly, $\Delta=\Delta^{\mathrm{re}} \cup \Delta^{\mathrm{im}}$. Let $\delta=\sum_{i=0}^{n} a_{i} \alpha_{i}$, where $a_{0}, a_{1}, \ldots, a_{n}$ are relatively prime integers and $A\left(a_{0}, a_{1}, \ldots, a_{n}\right)=0$. For all $i=0, \ldots, n$,

$$
r_{i}(\delta)=\delta-\delta\left(h_{i}\right) \alpha_{i}=\delta .
$$

So, $\delta \in \Delta^{\mathrm{im}}$. In fact, $\Delta^{\mathrm{im}}=\{k \delta \mid 0 \neq k \in \mathbb{Z}\}$.

The set of real roots $\Delta^{\text {re }}$ for the affine Lie algebra $\mathfrak{g}$ is

$$
\Delta^{\mathrm{re}}=\left\{\alpha+k \delta \mid \alpha \in \stackrel{o}{\Delta}_{s}, k \in \mathbb{Z}\right\} \cup\left\{\alpha+r k \delta \mid \alpha \in \stackrel{o}{\Delta}_{l}, k \in \mathbb{Z}\right\},
$$

if $\mathfrak{g}$ is not of type $A_{2 n}^{(2)}$,

or

$$
\begin{aligned}
\Delta^{\mathrm{re}}=\{ & \left.\frac{1}{2}(\alpha+(2 k-1) \delta) \mid \alpha \in \stackrel{o}{\Delta}_{l}, k \in \mathbb{Z}\right\} \cup\left\{\alpha+k \delta \mid \alpha \in \stackrel{o}{\Delta}_{s}, k \in \mathbb{Z}\right\} \\
& \cup\left\{\alpha+r k \delta \mid \alpha \in \stackrel{o}{\Delta}_{l}, k \in \mathbb{Z}\right\}, \quad \text { if } \mathfrak{g} \text { is of type } A_{2 n}^{(2)},
\end{aligned}
$$

where $\stackrel{o}{\Delta}_{l}$ and $\stackrel{o}{\Delta}_{s}$ denote the set of long and short roots, respectively, for the associated finite dimensional simple Lie algebra $\stackrel{o}{\mathfrak{g}}([4])$. We know that for all dominant integral $\lambda \in \tilde{t}^{*}$, there exists a unique (up to isomorphism), irreducible, integrable highest weight module, denoted by $L(\lambda)$. For the purpose of this paper and without loss of generality, we can and do assume that $\lambda(d)=0$. Thus,

$$
\lambda=\sum_{i=0}^{n} k_{i} \Lambda_{i}, \quad k_{i} \in \mathbb{Z}_{\geq 0}
$$

where $\Lambda_{0}, \ldots, \Lambda_{n}$ are the fundamental weights defined by $\Lambda_{i}\left(h_{j}\right)=\delta_{i j}$, and $\Lambda_{i}(d)=$ 0 . The canonical central element is $c=\sum a_{i}^{\prime} h_{i}$, where $a_{0}^{\prime}, \ldots, a_{n}^{\prime}$ are relatively prime integers such that $\left(a_{0}^{\prime}, \ldots, a_{n}^{\prime}\right) A=0$. Hence, $\lambda(c)$ is the level of $L(\lambda)$. 
Consider $\mathbb{Z}\left[\left[e\left(-\alpha_{0}\right), \ldots, e\left(-\alpha_{n}\right)\right]\right]$, the ring of formal power series in $e\left(-\alpha_{0}\right), \ldots$, $e\left(-\alpha_{n}\right)$, where $e(\cdot)$ denotes the formal exponential. We let $(s)=\left(s_{0}, \ldots, s_{n}\right) \in$ $\left(\mathbb{Z}^{+}\right)^{n}$ and define the $(s)$-specialization homomorphism to be

$$
f_{(s)}: e\left(-\alpha_{i}\right) \mapsto q^{s_{i}}, \quad i=0, \ldots, n .
$$

By definition, $\operatorname{mult}(\alpha)=\operatorname{dim}\left(\mathfrak{g}_{\alpha}\right)$. It is known that

$$
\operatorname{mult}(\alpha)=\left\{\begin{aligned}
1, & \alpha \in \Delta^{\mathrm{re}}, \\
\operatorname{rank}(A), & \alpha=k r \delta, \\
\frac{\mathrm{N}-\operatorname{rank}(A)}{r-1}, & \alpha=s \delta, s \neq_{r} 0,
\end{aligned}\right.
$$

where $\mathfrak{g}=X_{N}^{r}$ is the affine Lie algebra. If we let $\lambda=s_{0} \Lambda_{0}+\cdots+s_{n} \Lambda_{n}$, then the principal specialization of the character of $L(\lambda)$ is given by

$$
\chi(L(\lambda))=\chi(L)=\chi\left(s_{0}, \ldots, s_{n}\right)=\frac{\left(s_{0}+1, \ldots, s_{n}+1\right) \text {-specialization of } D^{v}}{(1, \ldots, 1) \text {-specialization of } D^{v}},
$$

where $D^{v}=\prod_{\alpha \in \Delta_{+}^{v}}(1-e(-\alpha))^{\operatorname{mult}(\alpha)}$, and $\Delta_{+}^{v}$ is the set of positive roots for $\mathfrak{g}^{v}$, the dual of $\mathfrak{g}$ (see [11]). This can be simplified further to

$$
\chi(L(\lambda))=\frac{f_{(s)+(1)}\left(D^{v}\right)}{f_{(1)}\left(D^{v}\right)},
$$

with $(1)=(1, \ldots, 1)$.

There exists an infinite dimensional Heisenberg subalgebra (see [7]), $\mathfrak{s}$, of $\mathfrak{g}$. The space $\Omega(L)$ of highest weight vectors in $L(\lambda)$ for $\underline{\mathfrak{s}}$ is the vacuum space for $L(\lambda)$. We need only calculate the principal character for $\Omega(L)$ since it completely determines $\chi(L(\lambda))$. It is known that

$$
\chi(\Omega(L))=\frac{\left(s_{0}+1, \ldots, s_{n}+1\right) \text {-specialization of } D^{v}}{[\varphi(q)]^{\operatorname{rank}(A)}}
$$

with $\varphi(q)=\prod_{k>0}\left(1-q^{k}\right)$. We denote $\chi(\Omega(L))$ by $\Omega\left(s_{0}, \ldots, s_{n}\right)$. The algorithm this paper describes calculates $\Omega\left(s_{0}, \ldots, s_{n}\right)$.

\section{Simplifying THE PROBLEM}

The core of the computation is the $\left(s_{0}+1, \ldots, s_{n}+1\right)$-specialization of $D^{v}$; the result is an infinite product over $k>0$ of a finite list of elements, each of the form $\left(1-q^{m k-b}\right)$. Each term corresponds to a root of the affine Lie algebra in the following sense: if $\alpha+k \delta$ is a root of the affine Lie algebra such that $e(-\delta)=q^{m}$ and $e(-\alpha)=q^{b}$, then that root generates the term $\left(1-q^{m k-b}\right)$. Furthermore, if $\alpha+k \delta$ generates $\left(1-q^{m k-b}\right)$, then $-\alpha+k \delta$ generates $\left(1-q^{m k+b}\right)$. Therefore, when generating our list of terms, we need only consider those generated by the positive roots, then use this symmetry to determine the terms generated by the negative roots.

The long roots of the associated finite dimensional simple Lie algebra generate roots of the form $\alpha+r k \delta$, as well as of the form $\frac{1}{2}(\alpha(2 k-1) \delta)$ if we are in $A_{2 n}^{(2)}$; the short roots of the associated finite dimensional simple Lie algebra generate roots of the form $\alpha+k \delta$ (2.1). If $r \neq 1$, the list of terms in any one character formula will have two different $k$ coefficients, $m$ and $r m$, where $e(-\delta)=q^{m}$. For uniformity and ease of computation, we will rewrite all the terms with respect to $\mathrm{rm}$. For example, if a formula has the term $\left(1-q^{5 k-1}\right)$ and the term $\left(1-q^{10 k-3}\right)$, we write 
$\left(1-q^{5 k-1}\right)$ as $\left(1-q^{10 k-1}\right)$ and $\left(1-q^{10 k-6}\right)$. In this way, each real root generates 1 or $r$ terms, and each imaginary root generates its multiplicity of terms. The key to the calulation is to determine how many terms are of the form $\left(1-q^{m k-b}\right)$, where $b=0, \ldots, m-1$. We will denote this by count $(b)$. This gives an expression of the form

$$
\prod_{k>0} \prod_{b=0}^{m-1}\left(1-q^{m k-b}\right)^{\operatorname{count}(b)} .
$$

Since $\varphi(q)=\prod_{k>0} \prod_{b=0}^{m-1}\left(1-q^{m k-b}\right)$, the final outcome will be

$$
\Omega\left(s_{0}, \ldots, s_{n}\right)=\prod_{k>0} \prod_{b=0}^{m-1}\left(1-q^{m k-b}\right)^{\operatorname{count}(b)-\operatorname{rank}(A)} .
$$

After getting the algebra and specialization from the user, we do the following: (1) generate the list of $b$ 's that corresponds to each of the roots, (2) count the number of occurrences for each $b$ to get count $(b)$, and (3) simplify the answer and return the principal character in the following form: $[\operatorname{count}(1), \operatorname{count}(2), \ldots, \operatorname{count}(m)]$. $($ Note: $\operatorname{count}(0)=\operatorname{count}(m)$.)

\section{Generating the List}

The first problem is to find the list of values, $b$, generated by the set of roots. The roots of the finite Lie algebra associated with the dual are linear combinations of the set of simple roots, $\left\{\alpha_{1}, \ldots, \alpha_{n}\right\}$. If $\alpha=\sum c_{\alpha, i} \alpha_{i}$, then in the $\left(s_{0}+1, \ldots, s_{n}+1\right)$ specialization,

$$
e(-\alpha)=q^{\sum_{i=1}^{n} c_{\alpha, i}\left(s_{i}+1\right)} .
$$

Thus, the value generated by $\alpha$ is $\sum_{i=1}^{n} c_{\alpha, i}\left(s_{i}+1\right)$. We call this value $b_{\alpha}$. To calculate all $b_{\alpha}$, we generate a coefficient matrix: $C=\left(c_{\alpha, i}\right)$. The order of the rows is unimportant. Thus, we have the product

$$
C\left[\begin{array}{c}
s_{0}+1 \\
\vdots \\
s_{n}+1
\end{array}\right]=B=\left(b_{\alpha}\right)_{\alpha \in \Delta_{+}} .
$$

Since $E_{6}, E_{7}, E_{8}, F_{4}$, and $G_{2}$ have a known set of roots (unlike the others that vary with $n$ ), their coefficient matrices can be generated using the Coxeter Maple package, written by John Stembridge of the University of Michigan ([13]), then stored. The other coefficient matrices can be generated as needed; this is fairly straightforward after noting the root patterns within each finite dimensional Lie algebra.

\section{Finding count $(b)$}

The second problem is to calculate $\operatorname{count}(b)$. We start by initializing a vector of length $r m$ to $\overrightarrow{0}$. We then traverse $\left(b_{\alpha}\right)_{\alpha \in \Delta_{+}}$, incrementing locations in this vector according to the value of $r$ and the type of the root $\alpha$. If $r=1$, then for each $b_{\alpha}$, we increment $\operatorname{count}\left(b_{\alpha}\right)$ and $\operatorname{count}\left(m-b_{\alpha}\right)$. If $r \neq 1$ and $\alpha$ is a long root, we increment $\operatorname{count}\left(b_{\alpha}\right)$ and $\operatorname{count}\left(r m-b_{\alpha}\right)$. If $r \neq 1$ and $\alpha$ is a short root, we increment $\operatorname{count}\left(b_{\alpha}\right), \operatorname{count}\left(r m-b_{\alpha}\right)$, and $\operatorname{count}\left(i m \pm b_{\alpha}\right), 0<i<r$. To take into account the imaginary root, we increment $\operatorname{count}(s m), s \not_{r} 0$ by $\frac{N-\operatorname{rank}(A)}{r-1}$ 
(see (2.1) $)$. Finally, we decrement each count $(b)$ by $\operatorname{rank}(A)$ which is equivalent to dividing by $[\varphi(q)]^{\operatorname{rank}(A)}$.

\section{Simplifying THE RESUlT}

The final problem is to simplify the formula. This step merely takes a vector and determines if it is a string of repeating subpatterns; if so, the subpattern is returned. For example, if the character formula generated is

$$
\prod_{k>0}\left(1-q^{10 k-1}\right)^{-1}\left(1-q^{10 k-4}\right)^{-1}\left(1-q^{10 k-6}\right)^{-1}\left(1-q^{10 k-9}\right)^{-1},
$$

the program should return

$$
\prod_{k>0}\left(1-q^{5 k-1}\right)^{-1}\left(1-q^{5 k-4}\right)^{-1}
$$

The first form is stored as the vector $[-1,0,0,-1,0,-1,0,0,-1,0]$ and the second as $[-1,0,0,-1,0]$.

\section{Results}

Calculating a principal character by hand is time-consuming and prone to error. My implementation of this algorithm calculates many formulas efficiently, thus making it easier to find patterns. It was clear to see that the only occurrences of the Rogers-Ramanujan products are those already found. This is proven below. Also, patterns in the level 2 and level 3 character formulas of $A_{2 n-1}^{(2)}$ were fairly obvious. All of the level 3 character formulas are proven as well as several of the level 2 character formulas. Patterns in $B_{n}^{(1)}, C_{n}^{(1)}$, and $D_{n}^{(1)}$ are conjectured but not proven.

7.1. The existence of the Rogers-Ramanujan identities in the principal characters of affine Lie algebras. For convenience, we denote the RogersRamanujan products by (cf. [1]):

$$
\begin{aligned}
& G(q)=\prod_{k>0}\left(1-q^{5 k-1}\right)^{-1}\left(1-q^{5 k-4}\right)^{-1}, \\
& H(q)=\prod_{k>0}\left(1-q^{5 k-2}\right)^{-1}\left(1-q^{5 k-3}\right)^{-1} .
\end{aligned}
$$

When referring to both simultaneously, $R(q)$ will be used.

It is known that the Rogers-Ramanujan products occur in the following affine Lie algebras: $A_{1}^{(1)}, A_{2}^{(1)}, A_{2}^{(2)}, A_{7}^{(2)}, C_{3}^{(1)}, F_{4}^{(1)}$, and $G_{2}^{(1)}([10])$.

To prove that these are the only occurrences of the Rogers-Ramanujan products in the principal characters of affine Lie algebras, we first note that to get a RogersRamanujan product, $e(-r \delta)=q^{5 \mu}$ where $\mu \in \mathbb{Z}^{+}$. This implies $r$ divides $\mu$. If there is a Rogers-Ramanujan product of the form $R\left(q^{p}\right)$, there exists $s$ such that $\mu=p s$. Therefore, there must be $(5 \mu-1) n-2 s$ terms to get a Rogers-Ramanujan product. 
Theorem 7.1. The only occurrences of the Rogers-Ramanujan products in the principal characters of affine Lie algebras are:

$$
\begin{aligned}
A_{1}^{(1):}: & \Omega(1,2)=\Omega(2,1)=G(q), \\
& \Omega(0,3)=\Omega(3,0)=H(q), \\
A_{2}^{(1)}:: & \Omega(1,1,0)=\Omega(0,1,1)=\Omega(1,0,1)=G(q), \\
& \Omega(2,0,0)=\Omega(0,2,0)=\Omega(0,0,2)=H(q), \\
A_{2}^{(2):}: & \Omega(2,0)=G\left(q^{2}\right), \\
& \Omega(0,1)=H\left(q^{2}\right), \\
A_{7}^{(2)}:: & \Omega(1,1,0,0,0)=G(q), \\
& \Omega(0,0,0,1,0)=H(q), \\
C_{3}^{(1)}: & \Omega(0,1,0,0)=\Omega(0,0,1,0)=G\left(q^{2}\right), \\
& \Omega(1,0,0,0)=\Omega(0,0,0,1)=H\left(q^{2}\right), \\
F_{4}^{(1)}:: & \Omega(0,0,0,0,1)=G\left(q^{4}\right), \\
& \Omega(1,0,0,0,0)=H\left(q^{4}\right), \\
G_{2}^{(1)}: \quad & \Omega(0,0,1)=G\left(q^{3}\right), \\
& \Omega(1,0,0)=H\left(q^{3}\right) .
\end{aligned}
$$

Proof. It is straightforward to calculate the number of terms generated by the roots of each algebra:

$$
\begin{aligned}
& A_{n}^{(1)}: n^{2}+n, \quad D_{4}^{(3)}: 12, \\
& A_{2 n-1}^{(2)}: 2 n^{2}, \quad E_{6}^{(1)}: 72, \\
& A_{2 n}^{(2)}: 4 n^{2}+3 n, \quad E_{7}^{(1)}: 126, \\
& B_{n}^{(1)}: 4 n^{2}-n-1, \quad E_{8}^{(1)}: 240 \text {, } \\
& C_{n}^{(1)} \quad: 2 n^{2}+2 n+1, \quad E_{6}^{(2)}: 48 \text {, } \\
& D_{n}^{(1)}: 2 n^{2}-2 n, \quad F_{4}^{(1)}: 74, \\
& D_{n+1}^{(2)}: 2 n^{2}, \quad G_{2}^{(1)}: 26 .
\end{aligned}
$$

So, to get a Rogers-Ramanujan product, we must find integers $n, \mu, p$, and $s$ such that the number of terms equals $(5 \mu-1) n-2 s$.

In $A_{n}^{(1)}$, we have

$$
\begin{gathered}
n^{2}+n=(5 \mu-1) n-2 s \\
n^{2}-(5 \mu-2) n+2 s=0 .
\end{gathered}
$$

Since $n \in \mathbb{N}$, the discriminant of the quadratic formula for $n$ must be a perfect square. Thus, there exists $z \in \mathbb{Z}$ such that

$$
(5 \mu-2)^{2}-8 s=z^{2} .
$$

If $\mu>2$, then $(5 \mu-2)^{2}>z^{2}>(5 \mu-3)^{2}$. Therefore, $\mu=1$ or 2 . If $\mu=2$, then $s=1$ or 2 , and in either case $n \notin \mathbb{N}$. If $\mu=1$, then $n=1$ or $n=2$, and we get the cases listed above for $A_{1}^{(1)}$ and $A_{2}^{(1)}$.

Similarly, for $A_{2 n-1}^{(2)}, A_{2 n}^{(2)}, B_{n}^{(1)}, C_{n}^{(1)}, D_{n}^{(1)}$, and $D_{n+1}^{(2)}$, we can find an upper bound on $\mu$ by manipulating the discriminant. We then look at the other values of 
$\mu$, which either lead to a contradiction or produce the Rogers-Ramanujan products we seek.

In $D_{4}^{(3)}$, we have

$$
\begin{gathered}
12=(5 \mu-1) 2-2 s, \\
7=s(5 p-1),
\end{gathered}
$$

which implies $s=1$ or 7 , but in each case, $p \notin \mathbb{N}$, so there are no Rogers-Ramanujan products in $D_{4}^{(3)}$.

Similarly, for $E_{6}^{(1)}, E_{7}^{(1)}, E_{8}^{(1)}, E_{6}^{(2)}, F_{4}^{(1)}$ and $G_{2}^{(1)}$, where the number of terms is fixed, we set that number equal to $(5 \mu-1) n-2 s$. This either leads to a contradiction or produces the Rogers-Ramanujan products we seek.

7.2. Level 2 character formulas in $A_{2 n-1}^{(2)}$. Since there is a diagram automorphism in $A_{2 n-1}^{(2)}$ that interchanges the $\alpha_{0}$ and $\alpha_{1}$ node, each of the following identities with $\Lambda_{0}$ can also be written with $\Lambda_{0}$ replaced with $\Lambda_{1}$. As the proofs are identical, these identities will not be explicitly stated.

Theorem 7.2. In $A_{2 n-1}^{(2)}$,

$$
\chi\left(\Omega\left(L\left(2 \Lambda_{0}\right)\right)\right)=\prod_{\substack{k \geq 1 \\ k \neq(n+1)}}\left(1-q^{2 k}\right)^{-1} .
$$

Proof. First, note that $e(-\delta)=q^{2(n+1)}$ and if $e(-(\alpha+k \delta))=q^{2(n+1) k+b}$, then $e(-(-\alpha+k \delta))=q^{2(n+1) k-b}$. Also, the multiplicity of $\left(1-q^{2(n+1) k}\right)$ is given in [4] to be $n$. Now it suffices to show that the number of $\alpha \in \Delta_{+}^{o}$ such that $e(-\alpha)=q^{b}$ or $e(-\alpha)=q^{2(n+1)-b}$ is $n$ if $b$ is odd and $n-1$ if $b$ is even.

Note that the roots in $\stackrel{o}{\Delta}_{+}$are one of two types: $\alpha_{i}+\cdots+\alpha_{j}, i \leq j$, or $\alpha_{i}+$ $\cdots+\alpha_{j-1}+2 \alpha_{j}+\cdots+2 \alpha_{n}, i<j$. When counting the number of $\alpha \in \stackrel{o}{\Delta_{+}}$such that $e(-\alpha)=q^{b}$, we consider the two different types of roots. In the case of roots of the form $\alpha_{i}+\cdots+\alpha_{j}$, the number of these roots such that $e(-\alpha)=q^{b}$ is $n+1-b$, assuming $b \leq n$. If $b>n$, then there are no roots of this form that give us $q^{b}$. In the case of roots of the form $\alpha_{i}+\cdots+\alpha_{j-1}+2 \alpha_{j}+\cdots+2 \alpha_{n}$, we count the number of partitions of $b$ of the form $(1, \ldots, 1,2, \ldots, 2)$ and of length at most $n$. The number of partitions of the above form is $\frac{b-2}{2}$ if $b$ is even and $\frac{b-1}{2}$ if $b$ is odd. If $b>n$, we subtract the number of extra partitions counted, which is $b-(n+1)$. Let $\operatorname{count}(b)^{+}$denote the number of $\alpha \in \stackrel{o}{\Delta_{+}}$such that $e(-\alpha)=q^{b}$. It is fairly straightforward to show that if $b$ is even, then count $(b)^{+}=\frac{2 n-b}{2}$, and if $b$ is odd, then $\operatorname{count}(b)^{+}=\frac{2 n+1-b}{2}$. If $b$ is even, then so is $2(n+1)-b$. So, the number of $\alpha \in \stackrel{o}{\Delta}$ + such that $e(-\alpha)=q^{b}$ or $e(-\alpha)=q^{2(n+1)-b}$ is

$$
\left(\frac{2 n-b}{2}\right)+\left(\frac{2 n-(2(n+1)-b)}{2}\right)=n-1 \text {. }
$$

If $b$ is odd, then so is $2(n+1)-b$. So, the number of $\alpha \in \stackrel{o}{\Delta_{+}}$such that $e(-\alpha)=q^{b}$ or $e(-\alpha)=q^{2(n+1)-b}$ is

$$
\left(\frac{2 n+1-b}{2}\right)+\left(\frac{2 n+1-(2(n+1)-b)}{2}\right)=n .
$$


Theorem 7.3. In $A_{2 n-1}^{(2)}$,

$$
\chi\left(\Omega\left(L\left(\Lambda_{0}+\Lambda_{1}\right)\right)\right)=\chi\left(\Omega\left(L\left(2 \Lambda_{0}\right)\right)\right) \prod_{k \equiv_{2(n+1)} \pm 1}\left(1-q^{k}\right)^{-1} \prod_{k \equiv_{2(n+1)} \pm 2}\left(1-q^{k}\right) .
$$

Proof. The difference between this character formula and $\chi\left(\Omega\left(L\left(2 \Lambda_{0}\right)\right)\right)$ is that $e\left(-\alpha_{1}\right)=q^{2}$ instead of $q^{1}$. For each root in $\stackrel{o}{\Delta}_{+}$that is affected, we have the following changes: (for ease of notation, let $b=-i$ refer to $b=2(n+1)-i$ )

$$
\begin{aligned}
\alpha_{1} & \Rightarrow-1 \text { to count }( \pm 1) \text { and }+1 \text { to count }( \pm 2), \\
\alpha_{1}+\alpha_{2} & \Rightarrow-1 \text { to count }( \pm 2) \text { and }+1 \text { to count }( \pm 3), \\
& \vdots \\
\alpha_{1}+\cdots+\alpha_{n} & \Rightarrow-1 \text { to } \operatorname{count}( \pm n) \text { and }+1 \text { to } \operatorname{count}( \pm(n+1)) .
\end{aligned}
$$

From roots of the form $\alpha_{i}+\cdots+\alpha_{j}$, we have a net gain of 1 in $\operatorname{count}( \pm(n+1))$ and a net loss of 1 in $\operatorname{count}( \pm 1)$.

From the roots of the form $\alpha_{i}+\cdots+\alpha_{j-1}+2 \alpha_{j}+\cdots+2 \alpha_{n}$, the only ones that are affected are $\alpha_{1}+2 \alpha_{2}+\cdots+2 \alpha_{n}, \ldots, \alpha_{1}+\cdots+\alpha_{n-1}+2 \alpha_{n}$. Using an argument similar to that used above, we find that these roots result in a net gain of 1 in count $( \pm 2)$ and a net loss of 1 in $\operatorname{count}( \pm(n+1))$.

Taking the two types of roots into account, we have a net gain of 1 in $\operatorname{count}( \pm 2)$ and a net loss of 1 in $\operatorname{count}( \pm 1)$.

Theorem 7.4. In $A_{2 n-1}^{(2)}$,

$$
\chi\left(\Omega\left(L\left(\Lambda_{2}\right)\right)\right)=\chi\left(\Omega\left(L\left(2 \Lambda_{0}\right)\right)\right) \prod_{k \equiv_{2(n+1)} \pm 2}\left(1-q^{k}\right)^{-1} \prod_{k \equiv_{2(n+1)} \pm 4}\left(1-q^{k}\right) .
$$

Proof. The difference between this character formula and $\chi\left(\Omega\left(L\left(2 \Lambda_{0}\right)\right)\right)$ is that $e\left(-\alpha_{2}\right)=q^{2}$ instead of $q^{1}$. Using a similar argument to that in the previous theorem, we find that the roots of the form $\alpha_{i}+\cdots+\alpha_{j}$ generate a net gain of 1 in count $( \pm n)$ and count $( \pm(n+1))$ and a net loss of 1 in count $( \pm 1)$ and count $( \pm 2)$. The roots of the form $\alpha_{i}+\cdots+\alpha_{j-1}+2 \alpha_{j}+\cdots+2 \alpha_{n}$ result in a net gain of 1 in count $( \pm 1)$ and count $( \pm(2(n-2)+3))$ and in a net loss of 1 in $\operatorname{count}( \pm(n+1))$ and count $( \pm n)$.

Taking both types of roots into account, we have a net gain of 1 in $\operatorname{count}( \pm 4)$ and a net loss of 1 in $\operatorname{count}( \pm 2)$.

This pattern seems to continue for all the level 2 character formulas for $A_{2 n-1}^{(2)}$.

Conjecture 7.1. In $A_{2 n-1}^{(2)}$, for $i=2,3, \ldots, n$,

$$
\chi\left(\Omega\left(L\left(\Lambda_{i}\right)\right)\right)=\chi\left(\Omega\left(L\left(2 \Lambda_{0}\right)\right)\right) \prod_{k \equiv_{2(n+1)} \pm i}\left(1-q^{k}\right)^{-1} \prod_{k \equiv_{2(n+1)} \pm 2 i}\left(1-q^{k}\right) .
$$

This holds true for $n=3, \ldots, 50$.

7.3. Level 3 character for $A_{2 n-1}^{(2)}$. Another interesting find was the generalized Rogers-Ramanujan products in the level 3 character formulas for $A_{2 n-1}^{(2)}$. At this point, it would be beneficial to add a bit of notation. Denote

$$
T^{ \pm}=\left\{ \pm\left(\alpha_{i}+\cdots+\alpha_{j}\right) \mid i \leq j\right\}
$$

and

$$
B^{ \pm}=\left\{ \pm\left(\alpha_{i}+\alpha_{i+1}+\cdots+\alpha_{j}+2 \alpha_{j+1}+\cdots+2 \alpha_{n}\right) \mid i<j\right\}
$$


So, $\stackrel{o}{\Delta}_{+}=T \cup B$, where $T=T^{+} \cup T^{-}$and $B=B^{+} \cup B^{-}$. For $b=1, \ldots, m-1$, let $S(b)=$ the number of $\alpha \in S$ such that $e(-(\alpha+k \delta))=q^{m k-b}$ when $e\left(-\alpha_{i}\right)=q$ for $i=1, \ldots, n$,

and

$$
\begin{gathered}
S(b, i)=\text { the number of } \alpha \in S \text { such that } e(-(\alpha+k \delta))=q^{m k-b} \\
\text { when } e\left(-\alpha_{j}\right)= \begin{cases}q, & j \neq i, \\
q^{2}, & j=i .\end{cases}
\end{gathered}
$$

Theorem 7.5. In $A_{2 n-1}^{(2)}$,

$$
\chi\left(\Omega\left(L\left(3 \Lambda_{0}\right)\right)\right)=\prod_{\substack{k>0 \\ k \neq 2_{2 n+3} 0, \pm 1}}\left(1-q^{k}\right)^{-1} .
$$

Proof. First, note that $e(-\delta)=q^{2 n+3}$ and the multiplicity of $\left(1-q^{2(n+1) k}\right)$ is given in [4] to be $n$. We need only show that

$$
\operatorname{count}(b)= \begin{cases}n-1, & b \neq 1,2 n+2 \\ n, & b=1,2 n+2\end{cases}
$$

for $b=1, \ldots, 2 n+2$. We know that

$$
T^{+}(b)= \begin{cases}n+1-b, & b<n+1 \\ 0, & b \geq n+1\end{cases}
$$

and that

$$
B^{+}(b)= \begin{cases}\frac{b-2}{2}, & b \text { even, } b \leq n+1 \\ \frac{b-1}{2}, & b \text { odd, } b \leq n+1 \\ n-\frac{b-2}{2}, & b \text { even, } b>n+1 \\ n-\frac{b-1}{2}, & b \text { odd, } b>n+1\end{cases}
$$

Furthermore,

$$
\stackrel{o}{\Delta}(b)=\operatorname{count}(b)=\stackrel{o}{\Delta}_{+}(b)+\stackrel{o}{\Delta}_{+}(2 n+3-b) .
$$

Also, $b$ and $2 n+3-b$ are both odd or both even. So

$$
\operatorname{count}(1)=\frac{2 n+1-1}{2}+0=n
$$

and

$$
\operatorname{count}(2 n+2)=\operatorname{count}(1)=n .
$$

For $b=2, \ldots, 2 n+1$, if $b$ is odd, then

$$
\operatorname{count}(b)=\frac{2 n+1-b}{2}+\frac{2 n-(2 n+3-b)}{2}=n-1,
$$

and if $b$ is even, then

$$
\operatorname{count}(b)=\frac{2 n-b}{2}+\frac{2 n+1-(2 n+3-b)}{2}=n-1 .
$$

Theorem 7.6. In $A_{2 n-1}^{(2)}$,

$$
\chi\left(\Omega\left(L\left(\Lambda_{0}+\Lambda_{n}\right)\right)\right)=\prod_{\substack{k>0 \\ k \neq \neq_{2 n+3} 0, \pm(2 n+1)}}\left(1-q^{k}\right)^{-1} .
$$


Proof. The difference between this character formula and $\chi\left(\Omega\left(L\left(3 \Lambda_{0}\right)\right)\right)$ is $e\left(\alpha_{n}\right)=$ $q^{2}$ instead of $q$. It suffices to show that

$$
\operatorname{count}(b)= \begin{cases}n-1, & b \neq 2,2 n+1 \\ n, & b=2,2 n+1 .\end{cases}
$$

The positive roots of the form $\alpha_{i}+\cdots+\alpha_{j}, i \leq j$, generate one less in count(1) and one more in $\operatorname{count}(n+1)$. Positive roots of the form $\alpha_{i}+\cdots+\alpha_{j-1}+2 \alpha_{j}+$ $\cdots+2 \alpha_{n}, i<j$, generate one less in $\operatorname{count}(b)$ if $2<b \leq n+1$ and one more in count $(b)$ if $n+2<b \leq 2 n+1$. If $b$ is odd,

$$
\stackrel{o}{\Delta}_{+}(b, n)= \begin{cases}n-\frac{b-1}{2}-1, & 1 \leq b \leq n \\ n-\frac{b-1}{2}, & n+1 \leq b \leq n+2, \\ n-\frac{b-1}{2}+1, & n+2<b \leq 2 n+1 .\end{cases}
$$

If $b$ is even,

$$
\stackrel{o}{\Delta}_{+}(b, n)= \begin{cases}n-1, & b=2, \\ n-\frac{b}{2}-1, & 3 \leq b \leq n, \\ n-\frac{b}{2}, & n+1 \leq b \leq n+2, \\ n-\frac{b}{2}+1, & n+2<b \leq 2 n+1 .\end{cases}
$$

Therefore,

$$
\operatorname{count}(b)= \begin{cases}n-1, & b \neq 2,2 n+1, \\ n, & b=2,2 n+1 .\end{cases}
$$

Theorem 7.7. In $A_{2 n-1}^{(2)}$, for $i=2, \ldots, n-1$,

$$
\chi\left(\Omega\left(L\left(2 \Lambda_{0}+\Lambda_{1}\right)\right)\right)=\prod_{\substack{k>0 \\ k \neq z_{2 n+3} 0, \pm 3}}\left(1-q^{k}\right)^{-1}
$$

and

$$
\chi\left(\Omega\left(L\left(\Lambda_{0}+\Lambda_{i}\right)\right)\right)=\prod_{\substack{k>0 \\ k \neq 2_{2 n+30, \pm(2 i+1)}}}\left(1-q^{k}\right)^{-1} .
$$

Proof. The difference between these characters and $\chi\left(\Omega\left(L\left(3 \Lambda_{0}\right)\right)\right)$ is that $e\left(\alpha_{i}\right)=$ $q^{2}, i=1, \ldots, n-1$, instead of $q$. It suffices to show that

$$
\operatorname{count}(b)= \begin{cases}n-1, & b \neq \pm(2 i+1) \bmod 2 \mathrm{n}+3 \\ n, & b= \pm(2 i+1) \bmod 2 \mathrm{n}+3\end{cases}
$$

For each of these character formulas, there is exactly one $i$ such that

$$
e\left(-\alpha_{j}\right)= \begin{cases}q, & j \neq i, \\ q^{2}, & j=i,\end{cases}
$$

where $i=1, \ldots, n-1$. Note that

$$
e\left(-\left( \pm \alpha_{i}+2 \alpha_{i+1}+\cdots+2 \alpha_{n}\right)\right)=q^{(2 n+3) k \mp(2 i+1)},
$$

so we need only show that if we exclude this root, the number of remaining roots that generate $b$ is $n-1$ for $b=1, \ldots, 2 n+2$. 
Comparing the differences with $\chi\left(\Omega\left(L\left(3 \Lambda_{0}\right)\right)\right)$, for $1 \leq i \leq \frac{n}{2}+1$, we see that

$$
T^{+}(r, w)= \begin{cases}T^{+}(r)-1, & 1 \leq r \leq i \\ T^{+}(r), & i<r \leq n-i+1, \\ T^{+}(r)+1, & n-i+1<r \leq n+1, \\ 0, & r>n+1\end{cases}
$$

and for $\frac{n}{2} \leq i \leq n-1$,

$$
T^{+}(b, i)=T^{+}(b, n+1-i) .
$$

For $1 \leq i \leq n-1$,

$$
\left(B^{+}-\left\{\alpha_{i}+2 \alpha_{i+1}+\cdots+2 \alpha_{n}\right\}\right)(b, i)= \begin{cases}B^{+}(b), & 1 \leq b \leq n+1-i \text { or } \\ & n+2 \leq b \leq 2 n+2-i, \\ B^{+}(b)+1, & 2 n+2-i<b<2 n+2, \\ B^{+}(b)-1, & n+1-i<b \leq n+1 .\end{cases}
$$

Therefore, for $1 \leq i \leq n-1$, if $b$ is even,

$$
\left(\stackrel{o}{\Delta}_{+}-\left\{\alpha_{i}+2 \alpha_{i+1}+\cdots+2 \alpha_{n}\right\}\right)(b, i)= \begin{cases}n-\frac{b}{2}-1, & 1 \leq b \leq i, \\ n-\frac{b}{2}, & i+1 \leq b \leq 2 n+2-i, \\ n-\frac{b}{2}+1, & 2 n+3-i \leq b \leq 2 n+2,\end{cases}
$$

and if $b$ is odd,

$$
\left(\stackrel{o}{\Delta}_{+}-\left\{\alpha_{i}+2 \alpha_{i+1}+\cdots+2 \alpha_{n}\right\}\right)(b, i)= \begin{cases}n-\frac{b-1}{2}-1, & 1 \leq b \leq i, \\ n-\frac{b-1}{2}, & i+1 \leq b \leq 2 n+2-i, \\ n-\frac{b-1}{2}+1, & 2 n+3-i \leq b \leq 2 n+2 .\end{cases}
$$

Thus,

$$
\left(\stackrel{o}{\Delta}-\left\{ \pm\left(\alpha_{i}+2 \alpha_{i+1}+\cdots+2 \alpha_{n}\right)\right\}\right)(b, i)=n-1, \quad b=1, \ldots, 2 n+2 .
$$

7.4. Further conjectures. Other interesting patterns seem to occur in $B_{n}^{(1)}, C_{n}^{(1)}$, and $D_{n}^{(1)}$.

Conjecture 7.2. In $B_{n}^{(1)}$,

$$
\chi\left(\Omega\left(L\left(\Lambda_{0}+\Lambda_{n}\right)\right)\right)=\prod_{k>0}\left(1-q^{2 k-1}\right)^{-1} .
$$

This holds for $n=3, \ldots, 50$.

Conjecture 7.3. In $C_{n}^{(1)}$,

$$
\chi\left(\Omega\left(L\left(\Lambda_{0}+\Lambda_{2}+\cdots+\Lambda_{n-1}\right)\right)\right)=\prod_{k>0}\left(1-q^{2 k-1}\right)^{-(n-1)} .
$$

This holds for $n=2, \ldots, 50$.

Conjecture 7.4. In $D_{n}^{(1)}$,

$$
\chi\left(\Omega\left(L\left(\Lambda_{0}+\Lambda_{n}\right)\right)\right)=\prod_{k>0}\left(1-q^{2 k-1}\right)^{-1} .
$$

This holds for $n=3, \ldots, 50$. 


\section{REFERENCES}

1. G. E. Andrews, The theory of partitions, in "Encyclopedia of Mathematics and Its Applications" (G. C. Rota, Ed.), Vol. 2, Addison-Wesley, Reading, Mass., 1976. MR 58:27738

2. M. K. Bos and K. C. Misra, Level Two Representations of $A_{7}^{(2)}$, Communications in Algebra 22 (1994), 3965-3983. MR 95d:17024

3. S. Capparelli, On some representations of twisted affine Lie algebras and combinatorial identities, J. Algebra 154 (1993), 335-355. MR 94d:17031

4. V. Kac, Infinite dimensional Lie algebras (3rd ed.), Cambridge Univ. Press, Cambridge, 1990. MR 92k:17038

5. J. Lepowsky and R. L. Wilson, Construction of the affine Lie algebra $A_{1}^{(1)}$, Comm. Math. Phys. 62 (1978), 43-53. MR 58:28089

6. J. Lepowsky and R. L. Wilson, A Lie-theoretic interpretation and proof of the RogersRamanujan identities, Adv. in Math. 45 (1982), 21-72. MR 84d:05021

7. J. Lepowsky and R. L. Wilson, The structure of standard modules. I. Universal algebras and the Rogers-Ramanujan identities, Invent. Math 77 (1984), 199-290. MR 85m:17008

8. M. Mandia, Structure of the level one standard modules for the affine Lie algebras $B_{l}^{(1)}, F_{4}^{(1)}$, and $G_{2}^{(1)}$, Mem. Amer. Math. Soc. 362 (1987). MR 88h:17023

9. A. Meurman and M. Primc, Annihilating ideals of standard modules of $s l(2, \mathbf{C})$ and combinatorial identities, Adv. in Math. 64 (1987), 177-240. MR 89c:17031

10. K. C. Misra, Specialized characters for affine Lie algebras and the Rogers-Ramanujan identities, in "Ramanujan revisited" (G. E. Andrews, et al., Ed), Academic Press, Inc., (1988). MR 89e:17019

11. K. C. Misra, Structure of certain standard modules for $A_{n}^{(1)}$ and the Rogers-Ramanujan identities, J. Algebra 88 (1984), 196-227. MR 85i:17018

12. K. C. Misra, Structure of some standard modules for $C_{n}^{(1)}$, J. Algebra 90 (1984), 385-409. MR 86h:17021

13. J. Stembridge, SF, posets, coxeter, and weyl, http://www.math.lsa.umich.edu/ jrs/maple. html\#coxeter. Accessed last on 19 July 1999.

14. C. Xie, Structure of the level two standard modules for the affine Lie algebra $A_{2}^{(2)}$, Comm. Algebra 18 (1990), 2397-2401. MR 91i:17039

Department of Mathematics, St. Lawrence University, Canton, New York 13617

E-mail address: mbos@stlawu.edu 